\title{
Editorial
}

\section{Urbane Ungleichheit in vergleichender Perspektive - Konzeptionelle Überlegungen und empirische Befunde aus den Americas}

\author{
E. Rothfuß ${ }^{1}$ and U. Gerhard ${ }^{2}$ \\ ${ }^{1}$ Geographisches Institut, Fakultät für Biologie, Chemie und Geowissenschaften, Lehrstuhl für Sozial- und \\ Bevölkerungsgeographie, Universität Bayreuth, Bayreuth, Deutschland \\ ${ }^{2}$ Geographisches Institut, Heidelberg Center for American Studies, Universität Heidelberg, \\ Heidelberg, Deutschland
}

Correspondence to: E. Rothfuß (rothfuss@uni-bayreuth.de) and U. Gerhard (ulrike.gerhard@geog.uni-heidelberg.de)

Received: 6 June 2013 - Revised: 5 September 2013 - Accepted: 13 September 2013 - Published: 22 July 2014

\begin{abstract}
Kurzfassung. Research on urban inequality has a long tradition in human geography as well as sociology. This special issue seeks to amplify the discussion by introducing some new theoretical approaches to the analysis. The first is to open up a research setting for comparative urbanism. By looking at urban life-worlds of marginalized neighbourhoods in the two Americas, the contributors do not want to search for similarities or disparities between different countries, but try to shed light on societal contexts and their spatial settings. The idea is to develop a reconstructive perspective to understand the uneven place-making within cities. With this, a second task is circumscribed: by describing and interpreting every-day life practices in Brazilian favelas and US ghettos, we want to contribute to a better understanding of patterns and spaces of urban inequality. Despite the wide array of (mostly quantitative) studies on urban inequality and segregation we declare a lack of understanding how these marginalized localities are experienced and reproduced. How do unprivileged inhabitants cope with everyday negligence and discrimination? Further concepts of urban citizenship, governmentality and the role of the penal state are introduced to enhance the conceptual as well as empirical analysis of inequality in cities.
\end{abstract}

\section{Einleitung}

Die Untersuchung von Armut und Ausgrenzung als Folgen urbaner Ungleichheit ist in der Stadtforschung nicht neu. Spätestens seit der Chicagoer Schule wird sozialräumliche Segregation als Merkmal von Stadtstrukturmodellen zur inneren Gliederung diskutiert. In jüngeren Ansätzen werden Segregation und Fragmentierung zwar anders begründet und deutlich kritischer bewertet (vgl. z.B. Dear und Flusty, 1998; Mollenkopf und Castells, 1991; SchneiderSliwa, 1996), dennoch als ein Charakteristikum der Gliederung des Stadtraumes angesehen. Während in den USamerikanischen Innenstädten das Ghetto das Synonym für Armut und Ungleichheit bildet, gelten in europäischen Städ- ten die in den 1960er/70er Jahre errichteten Großwohnsiedlungen als "Stadtteile mit besonderem Entwicklungsbedarf“ oder ,zone urbain prioritaire“ und somit als „Problemviertel“ (Brailich et al., 2008). Deren Marginalisierung wird zum Teil als Indikator einer „Amerikanisierung“ von Städten kontrovers verhandelt (z.B. Klagge, 2001; Häussermann et al., 2004; Wacquant, 2004; Murray, 2006; Lapeyronnie, 2009; Nobles, 2010; Garbin und Millington, 2012). Auch die Stadtforschung zu Lateinamerika hat eine lange Tradition zur Untersuchung segmentärer Urbanität (Bähr und Mertins, 1995; Wehrhahn, 1998; Fischer und Parnreiter, 2002; Coy, 2006; Deffner, 2010; Rothfuß, 2014), wobei die brasilianischen Favelas zu Synonymen für Marginalisierung und Exklusion der „Peripheren Moderne“ (Souza, 2008) wurden. 
Es stellt sich jedoch die Frage, ob eine separate Behandlung von Ungleichheit in Städten des Globalen Nordens und denen des Globalen Südens überhaupt sinnvoll ist (vgl. Wehrhahn, 2014; Gerhard, 2014). Besitzen US-amerikanische Ghettos nicht eher Parallelen zu den brasilianischen Favelas als zu den räumlich näheren anderen Stadtteilen nordamerikanischer Städte? Welche Rolle spielt der Staat, bzw. die Stadt-Staat-Beziehungen für Ausmaß und Bedeutung von Ungleichheit in der Stadt? Abgesehen von der Wichtigkeit von Untersuchungen zur Messung und Feststellung von Ungleichheit innerhalb von Städten gilt es unabhängig von einem jeweiligen nationalen Kontext zu untersuchen, wodurch sich die heutigen in Nord und Süd existierenden Lebenswelten der Marginalviertel auszeichnen, inwiefern sich die Strukturen der urbanen Ungleichheit reproduzieren. Es wird also eine komparative Perspektive gewählt, die nicht miteinander vergleicht, um Gemeinsamkeiten und Unterschiede herauszustellen oder gar zu messen, sondern um ein tieferes Verständnis zu entwickeln, wie Ungleichheit gesellschaftlich hergestellt und reproduziert wird sowie welche (kollektiven) Praktiken entwickelt werden, damit im Alltag zu verfahren.

Ziel des vorliegenden Beitrags wie auch des Themenheftes ist es, gesellschaftswissenschaftliche Stadtforschung in vergleichender Perspektive Raum zur Reflexion und Weiterentwicklung zu geben, um die verschiedenen Dimensionen von urbaner Ungleichheit zu verdeutlichen. Es geht also um eine Begründung dieser Forschungsperspektive sowie das Aufzeigen von Möglichkeiten einer konzeptionellen und empirischen Umsetzung.

\section{Gleichheit als politischer Grundsatz}

Der Diskurs um gesellschaftliche Ungleichheit geht zurück zu den Anfängen der politischen Philosophie der Antike. Dass Gesellschaften eine kontingent disparitäre Verteilung materieller (und immaterieller) Ressourcen aufweisen, fordert zu Gleichheit unter den Menschen als ein zentrales anzustrebendes politisches Ziel auf, das noch immer konstitutiv für die (alt-)europäische Politik ist. Es existiert gleichzeitig ein ebenso mächtiger Diskurs darüber - eher in den Americas sowie in Großbritannien - dass Ungleichheit immanenter Bestandteil (moderner) Gesellschaften sei und eine Verwirklichung der Gleichheit unter den Menschen schlicht unmöglich und daher eine bereits theoretisch unproduktive Forderung sei.

Ein Ringen um das „gute Leben“, das auch soziale Gerechtigkeit impliziert, ist somit ein fortwährender und utopischer Kampf. Es kann nicht als teleologischer Entwicklungsprozess missverstanden werden, denn in einer Diktatur der Gleichheit wird gerade der darin innewohnende Grundgedanke pervertiert und totalisiert: die „Phantasmagorie des Gerechtseins“"gegenüber den anderen bringt ihren eigenen diskriminatorischen Abgrund hervor (Dörfler, 2013, S. 42 in
Bezug auf Žižek, 2009, S. 80ff.). Dies kommt auch in der politischen Philosophie von Rancière (2002, S. 44) zum Ausdruck, der für eine konzeptionelle Beschäftigung mit urbaner Ungleichheit von bemerkenswerter Bedeutung sein sollte:

Die Gleichheit ist kein Gegebenes, das die Politik einer Anwendung zuführt, keine Wesenheit, die das Gesetz verkörpert, noch ein Ziel, das sie sich zu erreichen vornimmt: Sie ist nur eine Voraussetzung, die in den Praktiken, die sie ins Werk setzen, erkannt werden muss.

Rancière basiert seine Überlegungen darauf, dass das Motiv des „Schädlichen“ und „Nützlichen“ nicht in die Kategorien von ungerecht und gerecht zu überführen sei. Zwischen diesen beiden Übergangskategorien besteht ein fundamentaler Unterschied. Das Gerechte der Polis ist ein Zustand, in dem sich von dem Vorteil des Einen nicht ein Schaden des Anderen ableiten lasse:

Die Politik existiert, wenn die natürliche Ordnung der Herrschaft unterbrochen ist durch die Einrichtung eines Anteils der Anteilslosen. Diese Einrichtung ist das Ganze der Politik als spezifische Bindungsform. Sie definiert das Gemeinsame der Gemeinschaft als politische Gemeinschaft (...). AuBerhalb dieser Einrichtung gibt es keine Politik, nur Ordnung der Herrschaft und Unordnung der Revolte. (Rancière, 2002, S. 24)

Ohne dass Rancière (2002, S. 54) annimmt, dass alle Menschen gleich sind, attestiert er aber jedem Menschen in einer ähnlichen Situation und mit der Möglichkeit ausgestattet zu sein, sich in einem Prozess der „Ent-Identifizierung“ von den zugewiesenen Plätzen zu lösen und einen neuen Subjektraum zu eröffnen, wodurch Emanzipation praktiziert wird (vgl. auch Dikeç, 2001). Daher ist Gleichheit kein zu erreichendes Ziel für die Gesellschaft; es ist eher ein Effekt, dem politisches Handeln motivational zu Grunde liegt. Diejenigen, die „Anteil haben an nichts“ (Rancière, 2002, S. 22), werden sich nicht dauerhaft mit der Freiheit als leerem Eigentum zufrieden geben, sondern ihren Anteil einfordern. Daher geht von den Parias und Unterdrückten die politische Sprengkraft aus, da alleine sie die „Universalität des Politischen“ für sich einfordern können (vgl. auch Marcuse, 1972). Dies gilt auch für Falvela- oder/und GhettobewohnerInnen in Nord- und Südamerika gleichermaßen, deren unterschiedliche Praktiken mit Ungleichheit zu verfahren im vorliegenden Beitrag untersucht werden.

Die Universalität des Politischen ist somit ein Schlüsselbegriff rancièrschen Denkens. Er lehnt eine apriorische Universalität fundamentaler Regeln oder Vorstellungen ab. Für ihn ist die politische Universalität die einzige, zu der die Menschen Zugang haben. Diese ist keine abstrakte, idealistische und elitäre Solidarität, sondern Solidarität im Konflikthaften. Es steht somit der Dissens, der „Streithandel“ im Zentrum 
politischer Artikulation. Dies rührt daher, dass es in einer gesellschaftlichen Ordnung einige gibt, die einen Anteil an der Verteilung der Macht sowie der Güter und Ressourcen haben (etwa hinsichtlich der ökonomischen Stellung, Klassenlage, Bildung, Raumzugangs, etc.) und andere, die keinen haben, die „Anteilslosen“. Politik setzt nun dort an, wo diese Logik durchbrochen wird. Politik ist dann Politik, wenn eine Unterbrechung und Durchkreuzung der geordneten Verteilungslogik erfolgt.

\section{Einordnung in den aktuellen Forschungsstand zu urbaner Ungleichheit in der Geographie}

Betrachtet man die jüngere Behandlung von sozialer Ungleichheit in der Geographie, wird der Blick sehr deutlich auf Disparitäten in Städten gelenkt, mit zum Teil unterschiedlichen Schwerpunktsetzungen. In den 1970er und 80er Jahren dominierten quantitative und räumliche Ansätze, die sich dem Postulat der statistischen Objektivität zuwandten. Als wichtiger Meilenstein, dieses Paradigma aufzubrechen, können die Arbeiten von Harvey $(1973,1996)$ genannt werden, die das Thema weiteren marxistischen, aber auch feministischen und radikalgeographischen Zugängen öffneten und ungleiche geographische Entwicklung als ein konstitutives Moment der kapitalistischen Produktionsweise betrachteten. Smith (1994) tritt explizit für eine normative Positionierung der Geographie im Kontext sozialer Gerechtigkeit ein und gilt somit als ein Mitbegründer der "Welfare Geography“, während Soja (2010) grundlegende Theorien zur sozial gerechten Verteilung von Gütern betrachtet und sie an aktuellen Themen aus Los Angeles diskutiert. Das dialektische Verhältnis von Gleichheit zum Konzept der Differenz haben die Geographen Young (1990) und Dikeç (2001) zur Diskussion gestellt und damit ebenfalls einen wichtigen theoretischen Beitrag geleistet, das Gleichheitspostulat kritisch zu verhandeln.

Soziale Ungleichheit besitzt eine räumliche Dimension und materialisiert sich insbesondere in Städten. Zeitgenössische Debatten ranken dabei um die Rolle des urbanen Raumes als „,contested space“ in der Produktion und Reproduktion sozialer Ungleichheit im Kontext neoliberaler Umstrukturierungsprozesse (Heeg, 2008; Leitner et al., 2007, für den Kontext der lateinamerikanischen Stadtgeographie Stren, 1994). Hier lassen sich allerdings sehr unterschiedliche Perspektiven feststellen. Auffällig ist die stete Zunahme an quantitativen Untersuchungen, die von Studien über einzelne Städte (z.B. Darden et al., 2010; Walks, 2001) bzw. dem räumlichen Vergleich zwischen Städten (z.B. O'Connor et al., 2003; Bolton und Breau, 2012; Lukinbeal et al., 2012; de Janvfy und Sadoulet, 2000; Bernt 2012 für den lateinamerikanischen Kontext), bis hin zu Messungen verschiedener Faktoren von Ungleichheit im amerikanischen Städtesystem reichen (z.B. Chakravorty, 1996; Glaeser et al., 2009).
Dem gegenüber stehen ethnographische Ansätze, die einzelne Städte in den Mittelpunkt rücken und unter einer lebensweltlichen Perspektive untersuchen (z.B. Hamer, 2011 zu East St. Louis, oder Gillette Jr., 2005 zu Camden, New Jersey oder Anderson, 2012 zu New York). Zum Teil werden dabei auch Medien- und Diskursanalysen angewandt, um die Bedeutung von „Rasse“ und Ethnizität im Ungleichheitsdiskurs herauszustellen (vgl. Wade, 1997; Wilson, 2007, 2009). Gerade auch die lateinamerikanische Stadt wird aufgrund ihrer bewachten Wohnkomplexe als hoch segregativ eingestuft (z.B. Caldeira, 2000), wobei Probleme von urbaner Gewalt in den Forschungsfokus rücken (vgl. Wacquant, 2008c; Briceño-León und Zubillaga, 2002; Portes und Hoffmann, 2003).

Einen breiten Raum nimmt die Diskussion urbaner Ungleichheit bei den Arbeiten zu Gentrifizierung ein. Nachdem „Klassiker“ wie Marcuse (1985), Smith (1996) von Anfang an auf die Bedeutung der Verdrängung und den damit einhergehenden Polarisierungseffekt hingewiesen haben, lässt sich in jüngerer Zeit ein Wiederaufleben der Debatte beobachten (vgl. Slater, 2006; Lees et al., 2008; Bernt und Holm, 2009; Brenner et al., 2009; Davidson und Lees, 2010; Dörfler, 2011, für den lateinamerikanischen Kontext exemplarisch Jones und Varley, 1999; Bromely und Mackie, 2009). Allerdings gibt es auch hier widersprüchliche Argumente: So belegen zum einen Untersuchungen, dass auch die afroamerikanische Mittelklasse von Gentrifizierung profitiert und die ethnische Segregation demnach abgemildert habe (McKinnish et al., 2010; Patillo, 2005), andere Ansätze diskutieren, ob soziale Mischung überhaupt wünschenswert ist (vgl. Miciukiewicz et al., 2012; Lees, 2008). Somit spiegeln auch diese verschiedenen Ansätze die Komplexität von Gleichheit als politischem Grundsatz wider, der auch in diesem Aufsatz zur Disposition gestellt wird.

\section{Komparative Stadtforschung - Der Vergleich als „Entdeckungsreise“}

Welchen Mehrwert bringt nun die vergleichende Perspektive von urbaner Ungleichheit, insbesondere zwischen Nord- und Südamerika (vgl. Gerhard und Rothfuß, 2009)? Zwar wird die Bedeutung komparativer Stadtforschung häufig betont (z.B. Dear, 2005; Bourne, 2008, Nijman, 2007; Davis und Tajbakhsh, 2005; McFarlane und Robinson, 2012), sie wird jedoch aufgrund methodischer Fallstricke und konzeptionelltheoretischer Unschärfen nur selten wirklich ernsthaft unternommen (vgl. Kantor und Savitch, 2005; Ward, 2010). So hat insbesondere Robinson $(2002,2006,2010)$ mehrfach darauf hingewiesen, dass Stadt- und Entwicklungsforschung an einer konstruierten Trennung zwischen urbanem Norden (Theoriebezug) und urbanem Süden (Entwicklungsbezug) implizit wie explizit festhält, sich damit selbst blockiert und die jeweils kontextsensitiven Erkenntnisse weitgehend ignoriert. Ähnlich argumentiert McFarlane (2010, S. 728) 
indem er konstatiert, dass vergleichende Untersuchungen von Städten häufig am Riff des „Entwicklungsdeterminismus“ stranden, da Städte diskursiv auf den Typus ,entwickelt“, „sich entwickelnd“ oder ,unterentwickelt“ festgeschrieben würden.

Darüber hinaus besteht ein weiteres Spannungsfeld komparativer Stadtforschung. Einerseits scheint die quantitative Forschungslogik in aller Regel keinen epistemologischen Zweifel an der „Operation Called ,Vergleichen““ zu kennen (Matthes, 1992, S. 88); andererseits hegen qualitativ verfahrende ForscherInnen ein Ressentiment am komparativen Projekt, da sie in ihren häufig hermeneutisch rekonstruktiven Feldzugängen die Einzigartigkeit und historisch kontingente Einmaligkeit des idiographischen Kontextes in das Zentrum ihrer Begründung stellen. Hierbei werden Verallgemeinerungen, die immer abstrahierend und generalisierend sind, oftmals kritisch beäugt (vgl. auch Kantor und Savitch, 2005, S. 137).

Vergleiche können letztlich passend oder unpassend erscheinen. Nur müssen die Einheiten und Kriterien der Vergleichsoperation transparent gemacht werden. Insbesondere in einer „Ethnographie urbaner Praktiken“ (Hannerz, 1980) kann eine Zugangsmöglichkeit gesehen werden, die Vergleichskriterien nicht als intrinsische Qualitäten der untersuchten Praktiken zu betrachten (vgl. Schmidt, 2012, S. 28ff.). Entscheidend ist daher, eine analytische Konzeptionierung von Vergleichskriterien, die mit apriorischen Ähnlichkeiten (Klasse, „Rasse“ und Gender) bricht (vgl. Varela und Dhawan, 2010, S. 313). In einem praxeologischen Verständnis werden diese Phänomene daher nicht im konzeptionellen Sinne präkonstruiert, sondern müssen als Voraussetzungen und Resultate von Praktiken als Herstellungsleistungen (,,doing race“ vgl. Wilson, 2007; Markus und Moya, 2011 und ,doing gender“" vgl. Kessler und McKenna, 2001; Bronner, 2011) aufgefasst werden. Es geht also um ein entdeckendes und offenes qualitatives Verfahren des Vergleichens, indem die Konstruktions- und Konstitutionsleistungen der untersuchten Subjekte in ihrem Alltag einer wissenschaftlichen Rekonstruktion unterzogen werden. Nach Bourdieu (1979) geht es darum, im empirischen Feld herauszufinden, was in den ungleichen Milieus letztlich ,auf dem Spiel steht". Es gilt daher, Kategorien, die urbane Ungleichheit produzieren und reproduzieren, empirisch zu entdecken und mit Objekten, Stadträumen und/oder Praktiken zu konfrontieren. Erst im Anschluss erfolgt eine Rekapitulation und Ermittlung von Analogien und/oder Differenzen gesellschaftlichen und urbanen „Eigensinns“. Es sind dies jedoch nicht essentielle Merkmale der Vergleichsobjekte, sondern Aspekte, die Subjekte (Kollektive) in einer derartigen praxeologischen Perspektive zu erkennen geben, sprich in der Eigenlogik ihrer sozialen Praxis tun wollen oder müssen. Mit Hilfe eines komparativen Ansatzes gilt es also zu rekonstruieren, wie ungleiche gesellschaftliche Verhältnisse in der Erscheinungsform des Städtischen als soziale und räumliche Ordnungen entstehen, wie es zu Regelmäßigkeiten und Reproduktions- formen (Naturalisierungseffekten) sozialen und räumlichen Lebens kommt und wie diese gesellschaftliche Ordnung von den „Anteilslosen“ versucht wird zu unterhöhlen, wie diese also unvernehmlich mit Ungleichheit verfahren.

\section{Zur konzeptionellen Untersuchung von urbaner Ungleichheit und ihrer empirischen Implikationen}

Urbane Praktiken als konstitutive Herstellungsleistungen von Urbanität im habituellen Sinne sind eingebettet in objektive Strukturen der Gesellschaft, die (bestimmte) Subjekte und ihre Kollektivierungsformen (Lebensstile, Klassen, etc.) ermöglichen, andere verunmöglichen. Daher erscheint es ratsam, bevor eine Perspektivierung auf Praktiken erfolgt, das Konzept der „Citizenship“ zu reflektieren und auf die gesellschaftlichen Kontexte in den Americas anzuwenden, da hierin Dimensionen gesellschaftlicher Strukturierung sichtbar werden, die entscheidend das Alltagsleben prägen.

\subsection{Urban Citizenship}

Der Begriff der Citizenship drückt traditionell die Mitgliedschaft zu einer politischen Gemeinschaft, meist im Sinne eines Nationalstaates, aus. Die Idee der Staatsbürgerschaft existiert seit drei Jahrtausenden und beruft sich auf die Zugehörigkeit zur griechischen Polis, die somit die Rechte der BewohnerInnen in der Stadt gleichbedeutend mit dem Staat definiert (Castoriadis, 1990). Als ein solches auf den Staat bezogenes Verständnis hat sich das Konzept bis heute erhalten, ist aber im Laufe des 20. Jahrhunderts erweitert und uminterpretiert worden (vgl. Isin, 2002; Smith und McQuarrie, 2012). Heute versteht man darunter nicht nur die Staatszugehörigkeit und die damit verbundenen legalen Rechte und Pflichten, sondern bezieht sie auf verschiedene räumliche Kontexte und Maßstabsebenen, angefangen von der lokalen, über eine regionale oder nationale bis hin zur globalen Ebene (vgl. Guarnizo, 2012; Rossi und Vanolo, 2012; Gerhard, 2007). Purcell (2003, S. 567) spricht von einer „Restrukturierung“ der Citizenship im Kontext des globalen politisch-ökonomischen Wandels. Welche horizontalen Verbindungen bestehen innerhalb einer Gemeinschaft, die zu einer politischen Abgrenzung gegenüber einer anderen $\mathrm{Ge}-$ meinschaft ausreichen? Hervorgerufen werden solche Verbindungen zum Beispiel durch gemeinsame Erfahrungen, Wertvorstellungen und Ziele, die in bestimmten räumlichen Kontexten bestehen. Dies bedeutet auch, dass Citizenship sich nicht nur auf legale Rechte bezieht, sondern auch Ideen, Praktiken und Beziehungen umfasst, die eine bestimmte politische Subjektivität und ein relatives Verständnis von Citizenship ausdrücken (Ehrkamp und Leitner, 2003). Sie ist kein Endzustand, sondern ein Prozess, der durch politische und alltagsweltliche Praktiken des Durchkreuzens in bestimmten räumlichen Kontexten ständig neu ausgefochten wird (Staeheli, 2003; Rancière, 2002). 
Zentral für ein solches Verständnis von Citizenship ist der Stadtraum und seine aufeinander bezogenen sozialen Gruppen. Die Praktiken der Citizenship und der damit einhergehende Kampf um Mitgliedschaft in einer Gemeinschaft werden machtvoll geformt durch die besondere Geographie der Stadt; Städte sind sozusagen die ,strategischen Arenen für die Ausbildung von Citizenship“ (Holston und Appadurai, 1999, S. 2). Soziale Ungleichheit innerhalb dieser kann demnach ein Auslöser für den Kampf um Citizenship sein und bestimmte soziale und politische Praktiken hervorbringen. Es entwickelt sich eine urbane Citizenship, die von Lefebvre (1968) prominent mit einem „Recht auf Stadt“ umschrieben worden ist und seitdem vielfach diskutiert wird (vgl. Harvey, 2008). Damit ist ein „Recht auf urbanes Leben“ oder - noch allgemeiner - ein „Recht auf die Straße“ angesprochen, was so viel bedeutet wie das Recht aller BewohnerInnen auf Nichtausschluss am gesellschaftlichen, politischen und sozialen Leben.

Allerdings lässt uns Lefebvres Plädoyer für ein Recht auf Stadt mit einem gewissen Grad an (gewollter) Ratlosigkeit zurück. Es bleibt ein offener Appell, ein konfligierender Aushandlungsprozess bzw. eine große Vision und praktische Utopie einer allgemein zugänglichen, offenen Stadt, ohne apriorisch totalitär festzulegen, wie Urban Citizenship nun planerisch oder politisch umzusetzen wäre. Es ist eine radikale These, die die herrschende neoliberale Ordnung oder Geographie der Stadt bis in die Grundfesten in Frage stellt (Purcell, 2003). Neue Konzepte wie die ,insurgent citizenship“ (Holston, 2001) zeigen Möglichkeiten des Widerstands und der Rebellion im urbanen Kontext auf.

\subsection{Subcitizenship in brasilianischen und US-amerikanischen Städten}

Versteht man Staatsbürgerschaft in diesem erweiterten Rahmen und betrachtet somit die effektive Umsetzung in Form gleicher Rechte, Zugangsmöglichkeiten und anerkennender Wertschätzung (Honneth, 1992), lässt sich in ungleichen Stadträumen bzw. Gesellschaften der Amerikas eine Form zweigeteilter Staatsbürgerschaft konstatieren.

So gilt nach Souza (2006) für einen großen Teil der BrasilianerInnen - insbesondere betrifft dies die afrobrasilianische Bevölkerung - lediglich eine Art zweitklassiger Staatsbürgerschaft, die er als subcidadania bezeichnet. Demnach besitzen diese BürgerInnen zwar formal gleiche Rechte, diese werden aber institutionell nicht effektiv umgesetzt. Dies gilt auch für einen bedeutenden Anteil US-amerikanischer StaatsbürgerInnen, die räumlich oft als GhettobewohnerInnen abgegrenzt und gesellschaftlich mit dem höchst umstrittenen Begriff der „urbanen Unterklasse“ (Wilson, 1987) umschrieben werden. Jüngere Untersuchungen weisen dabei auf die anhaltende Bedeutung von „Rasse“ - nämlich der afroamerikanischen Bevölkerung - als wichtigstem Distinktionsmerkmal für Marginalisierung und Benachteiligung hin (vgl. z.B. Wacquant, 2002; Wilson, 2007, 2009).
Geht man den Ursachen dieser zweigeteilten Staatsbürgerlichkeit auf den Grund, lassen sich für den brasilianischen wie auch den US-amerikanischen Kontext interessante Analogien aber auch Differenzen beobachten. So liegt diese in den Augen von Souza (2006) für Lateinamerika in einem selektiven und unvollständigen Modernisierungsprozess begründet. Die komplexen peripher-modernen Gesellschaften, die sich im Zuge europäischer Kolonialexpansion herausgebildet haben, stellen einen neuen Gegentypus zu Europa, der „Zentralen Moderne“ dar. Sie haben den westlichen Rationalismus als soziale Formation erst mit den Einwirkungen europäischer Kolonisation erhalten, verinnerlicht und dadurch erst moderne Institutionen konstituiert. Es fehlte jedoch eine soziale Entwicklung durch die Aufklärung; Rationalisierungsprinzipien wurden ohne gesellschaftlichen Wandel implementiert, wodurch ein auf Gerechtigkeits- und Gleichheitsprinzipien basierender bürgerlicher Gefühlshaushalt in allen sozialen Klassen nahezu ausblieb. Die meisten Mitglieder der brasilianischen Mittel- und Oberklasse würden zwar niemals eine effektive soziale und juristische Nichtanerkennung der Unterklasse bestätigen, aber dennoch existiert ein stummer sozialer Konsens durch unsichtbare Netze von Klassensolidarität in Form tiefer subtiler Ressentiments gegenüber der Unterklasse.

Für die USA kann konstatiert werden, dass nach der Aufhebung der Rassentrennung in den 1960er Jahren das Gleichheitsprinzip nach und nach verankert und umgesetzt werden sollte. Am Beispiel des öffentlichen Diskurses über Gewalt im amerikanischen Ghetto vertritt Márquez (2012, S. 627) jedoch die These, dass das Rassenargument heute sehr viel komplexer und geradezu ,heimtückischer" als früher wirke. Da die Kolonialisten durch die europäische Aufklärung dem Gleichheitsprinzipien ganz explizit verbunden waren, erscheint „Rasse“ zum einen sozial konstruiert (klassische Ansätze), zum anderen aber auch in radikalerer Auslegung als ein neu zu definierendes ,Attribut europäischer Moderne“ (ebda.), das es ermöglicht, aktuelle Formen von polizeilicher Gewalt und sozioökonomischer Exklusion durchzusetzen bzw. zu legitimieren. So sind die kolonialen Strukturen noch in der heutigen Gesellschaft erkennbar, indem das Bild des ,,noblen/edlen Wilden“ - erzählt in der frühen amerikanischen Erzählung „Last of the Mohicans“ von James Fenimore Cooper - dem des ,brutalen, blutrünstigen Wilden“ gegenübergestellt wird. Übertragen auf den heutigen urbanen Raum könne man also unterscheiden zwischen denjenigen, die es (aus eigener Kraft) geschafft haben, dem urbanen Dschungel und damit den pathologischen Strukturen des Ghettos zu entkommen, gegenüber jenen, die es sich durch Gewalttaten, Delinquenz und/oder sonstiges persönliches Versagen im Ghetto „,bequem“ gemacht hätten und dem ,code of the street“ (Anderson, 1994) verhaftet geblieben seien. Einzelne afroamerikanische Erfolgsgeschichten - wie die von Präsident Barack Obama - verfestigen dieses Bild pathologischer Strukturen im Ghetto weiter, da sie aufzeigen, dass man diesen entkommen könne, wenn man 
sich nur genug anstrenge (Berg, 2003). Dieser leistungsideologische bürgerliche Diskurs existiert äquivalent in Brasilien. Die bürgerlichen Mittelschichten entlasten sich mit ihrem propagierten Arbeits- und Leistungsethos, ihrem verinnerlichten Willen zum Tun, der zum Erfolg führt, denn wer seine Möglichkeiten nutzt, wird dafür auch belohnt. Da im kapitalistischen Brasilien wie in den USA die Arbeit die zentrale gesellschaftliche Anerkennungskategorie darstellt, durch welche Identität, Respekt und gesellschaftliche Achtung gesichert werden können, wird von den Privilegierten eine Leistungsideologie propagiert, die zur Legitimierung ihres Herrschaftsanspruches dient (Rothfuß, 2012, S. 172ff.).

In den USA und in Brasilien entbehren die ,urban outcasts" (Wacquant, 2008a) positiver und anerkennender Wertung. Sie sind Staatsbürger zweiter Klasse, da sie im Verwertungsprozess und Konkurrenzkampf der Städte überflüssig sind und in der neoliberalen „trope“ (vgl. Beitrag von Wilson in diesem Heft) von Wachstum und Prosperität eine dysfunktionale, unproduktive und überflüssige Schattenbevölkerung darstellen. Wenn also der Zustand einer persistenten Subcitizenship nicht über eine Politik der Gerechtigkeit aufgehoben werden kann, werden es die Anteilslosen selbst sein (müssen), die durch Praktiken des Widerstandes und der Selbstermächtigung ihren Anteil einfordern. Dazu sind Taktiken im Alltagsleben relevant, auf die im anschließenden Kapitel fallspezifisch und komparativ eingegangen wird.

\subsection{Urbanes Alltagsleben}

Im Alltagsleben, wie es etwa von Lefebvre (1974) und De Certeau (1988) konzeptionalisiert wird, stehen die „Geographien des Möglichen“ der „Schwachen“ im Vordergrund. Diese sind aber nicht von der herrschenden Ordnung zu trennen. So liegt De Certeaus (1988, S. 20) Ausgangspunkt, kritische Gesellschaftstheorie zu betreiben, in der Annäherung an das Alltagsleben, um dabei die Frage der Machtverhältnisse zwischen herrschender Ordnung und den sie protegierenden Strukturen einerseits und der ,schweigenden Mehrheit" andererseits zu klären. Damit ist De Certeaus ,move to the margins", wie es Poster (1992, S. 101) formuliert hat, eine Verlagerung vom Feld der Macht hin zu den partikularen und kontextgebundenen Praktiken des Alltags. Er thematisiert Formen der Vergemeinschaftung, über die sich die Subjekte in ihrer eigenen Lebenswelt verorten. Der ,gemeine Mann“ oder der „Held des Alltags“ (De Certeau, 1988, S. 9) macht seine eigene Geschichte, macht seine eigene und mögliche Geographie unter Konditionen, die er jedoch nicht selber wählen kann. Diese Marxsche Maxime drückt De Certeau (1988, S. 60) in der Expression des Mitmachens aus: „Man muss ,mitmachen", indem man etwas damit macht“. Er richtet sich daher auf die ,abertausend Praktiken“ (ebd., S. 16), die den Alltag konstituieren.

Analog zu einer Geographie des Möglichen bedeutet auch bei Lefebvre (1974, S. 511ff.) das Alltagsleben nicht nur eine kapitalistisch beschädigte Alltagspraxis, welche die ge- sellschaftlichen Verhältnisse als gegeben hinnimmt und damit gesellschaftliche Kontinuität der Herrschenden (derer die Anteil haben an Etwas) garantiert, sondern sie ist auch der „Ort des Möglichen“. Dieser Raum, in dem die Spontanität, das Schöpferische und die Kreativität durch ihre Verdinglichung verstummt sind, ist anhand von Praktiken der Aneignung, der Umwidmung und „Entdinglichung“ wieder sichtbar zu machen (vgl. auch Maffesoli, 1986). Insofern steht bei Lefebvre einer Kritik des Alltagslebens dialektisch auch deren Rehabilitierung gegenüber, die auf den Referenzpunkt des ,totalen Menschen“ abzielt (vgl. Schmid, 2005). Da im ,abstrakten Raum“ von Lefebvre (1974) grundsätzlich das Paradigma einer marktgerechten Verwendbarkeit von Raum, seiner Quantifizierbarkeit und bürokratischen Verwaltung und Kontrolle vorherrscht, sind Räume des Widerstandes (im „,gelebten“ und „differentiellen“ Raum) mit schöpferischen Alltagspraktiken seiner Bewohner rar.

De Certeau möchte der Widerständigkeit populärer Praktiken Rechnung tragen, ohne den kritischen Blick auf die disziplinierende und gouvernementale Macht der herrschenden Verhältnisse auszublenden. Um dieses Spannungsfeld konzeptionell austarieren zu können, liefert er ein entsprechendes analytisches Modell, das eine Unterscheidung von Strategien und Taktiken vornimmt. Hierzu formuliert De Certeau (1988, S. 87): „Als Strategie bezeichne ich die Berechnung (oder Manipulation) von Kräfteverhältnissen, die in dem Moment möglich wird, wenn ein mit Willen und Macht versehenes Subjekt (ein Unternehmen, eine Armee, eine Stadt oder eine wissenschaftliche Institution) ausmachbar ist. Sie setzt einen Ort voraus, der als etwas Eigenes beschrieben werden kann (...).“ Im Gegensatz zu den Strategien (...) bezeichnet De Certeau (1988, S. 89) als Taktik ein „Handeln aus Berechnung, das durch das Fehlen von etwas Eigenem bestimmt ist. Keine Abgrenzung einer Exteriorität liefert ihr also die Bedingungen einer Autonomie. Die Taktik hat nur den Ort des Anderen. Sie muss mit dem Terrain fertig werden, das ihr so vorgegeben wird, wie es das Gesetz einer fremden Gewalt organisiert (...): sie ist eine Bewegung, innerhalb des Sichtfeldes des Feindes, (...) die sich in einem von ihm kontrollierten Raum abspielt. (...) sie macht einen Schritt nach dem anderen. Sie profitiert von ,Gelegenheiten ' und ist von ihnen abhängig." Die Schwachen sind damit die Taktierer. Ihre Taktiken oder „Coups“ (De Certeau, 1988, S. 31) gegenüber den Starken sind dabei aber immer ephemer, flüchtig und auch unsichtbar.

Die hier vorgeschlagene Perspektivierung auf Strategien und Taktiken kann als eine heuristische Möglichkeit betrachtet werden, urbane Ungleichheit empirisch zu erforschen und herauszuarbeiten, welche Praktiken der Ausschließung von Seiten der Starken und den taktischen Reaktionen und Widerständigkeiten der Schwachen in entsprechenden Sozialwelten von Nord- und Südamerika alltäglich hergestellt werden. 


\subsection{Alltagspraktiken als Taktiken in Favela und Ghetto}

Versucht man, urbane Alltagspraktiken in Favela und Ghetto empirisch zu deuten, lassen sich in einer ersten Interpretation basierend auf eigenen Forschungen drei Typen als Analysekriterien unterscheiden.

\subsubsection{Praktiken der pragmatischen Resignation}

Die Benachteiligten in den USA und Brasilien besitzen durchaus ein Unrechtsempfinden und eine moralische Urteilskraft darüber, was gerecht ist und was nicht. Auch haben sie ein genaues Bewusstsein über die begrenzten Möglichkeiten des Opponierens und der Auflehnung gegen ungerechte gesellschaftliche Verhältnisse. Daraus wäre teilweise der Schluss zu ziehen, dass die moralische Urteilskraft der Ghetto- und FavelabewohnerInnen dazu führen kann, dass diese pragmatisch und im Grunde rational mit der in ihren Augen unüberwindlichen sozialen Ungleichheit verfahren. Die ungerechte Klassenstratigraphie zu akzeptieren, meint nicht zwangsläufig, alles hinzunehmen bzw. in Agonie zu verfallen. Diese Haltung beschreibt Scott (1985, S. 325) als ,attitude of pragmatic resignation“. Sie zeigt sich sehr deutlich in den beiden Zitaten aus Favela und Ghetto:

[...] Was können wir denn tun gegen das ungerechte System? Also ignorieren wir die Reichen genauso wie sie uns ignorieren. Die Welt der Reichen interessiert uns nicht. (Favelabewohner aus Calabar, Salvador, Interview August 2006, E. Rothfuß)

Don't wait around for nobody. A man can't wait for somebody else to protect his kids and his family; he got to move on that his own self. (zitiert in Hamer, 2011:177)

Indem die Anderen für nicht relevant erklärt werden, gelingt den BewohnerInnen eine kollektiv geteilte Selbstbezüglichkeit. In der erlittenen Verweigerung von Anerkennung vollbürgerlicher Rechte etablieren sich in der Unterklasse Abgrenzungsweisen, die sich in einer abgrenzenden Identitätskonstruktion als eine Art Selbstversicherung dokumentieren und durch die Abwertung der privilegierten Gegenwelt und Aufwertung der eigenen Lebenswelt gekennzeichnet ist (vgl. Rothfuß, 2012). So beschreibt eine Favelabewohnerin, ebenfalls aus Salvador:

Die Welt der Reichen ist doch schlecht. (...) Die sind reich, aber krank. Haben so viele Sorgen um ihr Geld, dass sie sich einbunkern müssen und alle Depressionen bekommen. Die dort [in den Hochhäusern] haben doch keine Lebensfreude. Die möchte ich nicht eintauschen gegen schicke Autos, bessere Kleidung, eine größere Wohnung. Ich brauche keinen Luxus. (Interview Januar 2007, E. Rothfuß)
Ganz ähnlich klingen die Worte eines Bewohners aus South Stockton, dem Hyperghetto der nordkalifornischen Stadt Stockton, der sich mit dem stigmatisierten Umfeld seiner Nachbarschaft abgefunden hat, indem er konstatiert, dass es in den ,besseren“ Stadtteilen auf eine andere Art und Weise sehr viel gefährlicher wäre zu leben:

I think I'd never move from my neighborhood. (...) And I think if I would to move on the other side of the neighborhood, like I said on the North Side there are still bad people and the worst thing is that if you are wealthy and those bad people know you are wealthy you are bad off. Basically you are always going to be robbed; you are always going to be harassed. Something is going to be... bad with you, but here where I live I already know how it is. I already experienced it and I understand how it is here. (Bewohner von Stockton, Interview April 2012, M. Holler, U. Gerhard)

Darin artikuliert sich Identität mit dem Eigenen und eine Herstellung von Sinn bezüglich der eigenen Lebenswelt. Die Favela bzw. das Ghetto wird zum klassenbezogenen Identifikations- und Anerkennungsraum, was auch nachfolgendes Zitat illustriert:

Eigenartige Welt dort. (...) Die haben alles, wissen nicht wohin mit ihrem Geld und werden krank davon. Die Menschen dort in den Hochhäusern leben wie in einem selbst gewählten Gefängnis. Die wirken doch irgendwie leblos und haben doch keine Lebensfreude. (Interview März 2006, E. Rothfuß)

Es zeigt sich hier eine ganz andere Perspektive als zum hegemonialen Stigmatisierungsdiskurs über Gewalt, Unsicherheit und Prekarität in Ghetto und Favela (vgl. Wacquant, 2007, 2008b; de Souza, 2012). Die aseptischen Welten der „Gated Communties“, ihre räumliche Hermetik und ihr Kontrollzwang aus Angst vor Überfällen etc. wird von den benachteiligten BewohnerInnen genau wahrgenommen und auf eine eigene Art und Weise interpretiert: Dem räumlich angrenzenden Bürgertum werden seine Kleinkariertheit und selbst verursachten Angstneurosen vorgehalten. Dies deutet darauf hin, dass die Subalternen ihre eigene Integrität und Würde zumindest teilweise wahren können. Dies sind die „Waffen der Schwachen“ (Scott, 1985), die hier im städtischen Kontext der Armen listig und selbstbewusst aufscheinen (vgl. Rothfuß, 2012, S. 226ff.).

\subsubsection{Kreative und kollektive Praktiken}

Nachbarschaftliche Beziehungen und Bindungen werden als wichtiges Argument für eine „funktionierende Nachbarschaft" angeführt. Gleichzeitig lasten territoriale Stigmata auf den benachteiligten Stadtvierteln, in denen Angst und Gewalt vorherrsche und niemandem mehr zu trauen sei. Die 
Perspektive der Bewohner zeigt jedoch eine interessante andere Sichtweise, im Folgenden erzählt von einer Bewohnerin aus Anacostia, Washington, D.C., dem ärmsten Stadtteil Washingtons, südöstlich des US Kapitols gelegen. Als ehemalige Krankenschwester einer Grundschule in Anacostia eröffnete sie hier einen Coffee Shop um die desperate Infrastruktur des Stadtteils zu verbessern - und diese ökonomisch und kreativ für sich zu nutzen:

I realized there was nowhere in this community for me to get a cup of coffee (...). I thought it would be wise to open something for the community here, and I wanted to make history by opening the first coffee shop in Anacostia. (www.washingtonpost. com)

So wirbt das Lokal, umgeben von sozialem Brachland, gar auf seiner Homepage damit, der erste und einzige Coffeeshop „East of the River“ (gemeint ist der Anacostia Fluss) zu sein. Aus einer Situation des Mangels wird durch Kreativität Einkommen, Existenz und Selbstermächtigung geschaffen. Auch wenn gleichzeitig die strukturellen Ungleichheiten bestehen bleiben, schafft diese eine von abertausenden Praktiken mit eine Grundlage für eine zukünftig gerechtere Gesellschaft.

Auch die BewohnerInnen der Favelas sind sich im Klaren darüber, dass nur sie ihre eigenen Lebensräume, ihre eigenen Wohnhäuser und auch den öffentlichen Raum selber zu gestalten und aufzubauen haben. Auf staatliche Hilfe kann dort wie in den vernachlässigten US-amerikanischen Stadtvierteln nicht ernsthaft gesetzt werden. Es geschieht weithin im kollektiven Tun des „,mutirão“, der Nachbarschaftshilfe. Denn die Favela hat keinen vorgegebenen Plan. Sie wurde nicht von ArchitektInnen entworfen und von BauherrInnen errichtet, sondern hat sich über die Alltäglichkeit des Begehens, des Verweilens und Zusammenlebens strukturell und lebensweltlich herausgebildet. Sie ist ein exklusives Kollektivprodukt der in ihr lebenden Menschen. Es geht in den konsolidierten Favelas heute also nicht mehr um kollektive Widerstandskämpfe eines Existenz- und Bleiberechts, sondern um die alltäglichen Kämpfe, von den urbanen Ressourcen unter spätkapitalistischen Bedingungen zu profitieren.

\subsubsection{Verdeckte und offene Widerständigkeit}

Schwarz sein, das bedeutet stark zu sein, oder? Widerstand zu leisten, zu kämpfen. (Sozialarbeiter in Calabar, Salvador; Interview September 2007, E. Rothfuß)

Die Formen und Artikulationen von Protest und Widerstand gegen rassistische Diskriminierung sind überaus vielfältig. Sie sind einerseits offen und zeigen sich als Gehorsamsverweigerung (z.B. Streiks, Proteste, Aufstände oder auch Randale). Sie können sich andererseits aber auch als alltäglicher Widerstand im Sinne einer vorgeblichen Gehorsamkeit ausdrücken, etwa in Form von Verzögerungstaktiken, kleinen
Diebstählen, schlechter oder falscher Ausführung von Arbeit oder auch in Form von Sabotage (vgl. auch Scott, 1985, S. 304ff.), hier als offene und verdeckte Widerständigkeit kategorisiert. Relevant ist dabei die Handlungsabsicht der widerständigen Akteure und nicht das Resultat der Praktik. Die Intention alltäglicher Widerstandsformen liegt darin begründet, dass diese Praktiken nicht „,bewiesen“ werden können und ihnen daher keine Sanktion folgt.

Ich arbeite hier als Rezeptionistin in einem Krankenhaus, hier in der Nähe von Calabar, für einen einzigen Mindestlohn im Monat. Monat für Monat. Warum soll ich hier arbeiten wie nochmal was? Da man meinen Bildschirm vom Computer nicht sehen kann, gehe ich ständig ins Internet, in Orkut und tausche mich mit meinen Freundinnen, Freunden und meiner Familie darüber aus. (Interview Januar 2007, E. Rothfuß)

Wie das Zitat zeigt, ist dies natürlich kein besonders ehrwürdiges Verhalten, sondern eher eines der ,wenigen verfügbaren Mittel einer untergeordneten Klasse, die Taten des Widerstands in eine sichere Verkleidung zu verpacken" (Scott, 1985, S. 78). Diesen offenen und alltäglichen Widerständigkeiten liegt eine ,,intuitiv gegebene Gerechtigkeitsvorstellung" (Honneth, 1992, S. 99) und damit ein Unrechtsempfinden der Subjekte zugrunde, die die alltäglichen Erfahrungen von Verletzung, Demütigung und Anerkennungsverweigerung erfahren müssen. Da sich dieser versteckte Müßiggang nicht kontrollieren lässt, kann er als ein „,faire de la perruque“ (De Certeau, 1988, S. 71) bezeichnet werden. Eine Perücke zu mimen ist eine Metapher für den Freiraum der Unterdrückten und Abhängigen. Es geht darum, aus dem Bestehenden taktisch Gewinn zu ziehen, ohne die gängigen Formen der Unterordnung in Frage zu stellen.

Ein Beispiel für eine mal eher verdeckte, mal aber auch bewusst offene Form von Widerständigkeit sind auch so genannte ,ghettowalks“. Hier wird die scheinbar alltägliche Form des Gehens zu einer Botschaft, zu einer unverfänglichen Taktik des Widerstands, indem Einzelne oder Gruppen von Bewohnern ein geographisches Feld im urbanen Machtgefüge abstecken. So schreibt Wilson (2007, S. 133) in seiner Analyse des US-amerikanischen Ghettos: „Such walking, far from being a delusional act of re-presentation, is a creative and nourishing re-reading of reality." Gerade die Gangs der Jugendlichen würden somit ihre Territorien abstecken und damit die hegemoniale räumliche Form herausfordern (ebda.), zugleich auch eine informelle Ökonomie aufbauen, die letztendlich sogar Aufstiegsmöglichkeiten und Jobgelegenheiten innerhalb dieser „Subwirtschaft“ bietet. Offenere Formen des Taktierens in Form von Gehen sind gemeinschaftliche Märsche von Politikern oder ,community group leaders“, die als öffentliche Veranstaltung fungieren und als Gang durch die Nachbarschaft ein Bewusstsein für diese Räume schaffen sollen. Solche Fußmärsche sind nur 
schwierig zu kontrollieren, selten aber ,unschuldig“ - und somit eine Kollektivpraktik der Widerständigkeit.

\section{Ausblick und die Beiträge in diesem Heft}

Der vorliegende Beitrag stellte den Vergleich der beiden urbanen Americas in den Mittelpunkt der Betrachtung, um die Ausprägung segregierter und ungerechter Lebenswelten aufzudecken. Diese Vorgehensweise beruht auf einer dezidierten Überzeugung, dass nord- und südamerikanische Städte ähnliche historische und gesellschaftliche Rahmenbedingungen besitzen, die alltagsweltlich nach wie vor eine opake aber wirkungsvolle Rolle spielen und damit auch subalterne Staatsbürger (,subcitizenship“) hervorbringen, die (sehr häufig) aufgrund ihrer Hautfarbe nach wie vor diskriminiert und stigmatisiert werden. In den Americas existiert eine geteilte bürgerliche Ideologie der Leistung, die dem Einzelnen sein Schicksal aufbürdet und damit von den strukturellen Ursachen sozialer Ungleichheit abzulenken vermag.

Hieraus leitet sich ein zweites Anliegen ab: mit einem Verständnis von den Alltagspraktiken in Favela und Ghetto lässt sich ein Beitrag zur Erforschung urbaner Ungleichheitsmuster und -prozesse leisten. Die Vielzahl von quantitativen Untersuchungen zu Ungleichheit in Städten ist sicherlich wichtig, um strukturelle und verteilungsdifferentielle Missstände aufzudecken, sie leisten aber noch keinen Beitrag dazu, wie die Lebensverhältnisse in diesen ungleichen Nachbarschaften wirklich sind, was sie für die Betroffenen bedeuten und wieso sie sich reproduktiv verfestigen. Indem wir eine Perspektive des Alltagslebens wählen, uns in ethnographischer Weise der urbanen Wirklichkeit zuwenden, halten wir uns das Soziale nicht vom Leib, sondern halten diese Art der relationalen Forschung für essentiell, um überhaupt angemessen die Sozialwelt rekonstruktiv begreifen und wissenschaftlich reflektieren zu können (Dörfler und Rothfuß, 2013).

Die exemplarisch gegenübergestellten Aussagen zur Lebenswirklichkeit in Washington, Stockton und Salvador da Bahia zeigten, dass die von Exklusion betroffenen Menschen z.T. ganz ähnliche Wahrnehmungs- Bewertungs- und Handlungsmuster aufwiesen um mit urbaner Ungleichheit zu verfahren. Es wurde aber auch deutlich, dass ein systematisches Forschungsprogramm apriorisch notwendig ist, um daraus sozial reale Feldlogiken im bourdieuschen Sinne zu generieren, die einer echten Komparatistik standhalten.

Der hier unternommene präliminare Versuch soll einen ersten Grundstein legen und befördert die Gewissheit, dass in den kapitalistischen Gesellschaften der Americas nichts dem Zufall überlassen ist: Will heißen, dass die Produktion und Reproduktion urbaner Ungleichheit einer (verborgenen) sozialen Grammatik folgt, die empirisch untersucht werden muss. In diesem Sinne tritt die hier vorgestellte komparative Perspektive an, um die wirklichen (im Sinne wirksamer) Gründe ungleicher Stadtentwicklung herauszuarbeiten. Die- ses Anliegen verfolgen auch die zwei weiteren Beiträge in diesem Themenheft.

So thematisiert Loï Wacquant die historischen Entwicklungsbedingungen und die Bedeutung des Begriffes Ghetto. Daraus wird die Notwendigkeit einer komparativen Perspektive USA - Europa - Südamerika abgeleitet und am Beispiel des Vergleichs US-amerikanischer Ghettos und französischer Banlieus ausgearbeitet. Ergebnis der Analyse ist, dass es nur wenige Parallelen zwischen den beiden Marginalvierteln gibt, da es sich vielmehr um zwei sehr unterschiedliche Formen handelt: das „Hyperghetto“ auf der einen, und das „Anti-Ghetto“ auf der anderen Seite. Eine wichtige These ist zudem die Rolle des Staates bei der Produktion von Ausgrenzung. Wacquant verwendet daher in seinem Beitrag bewusst den Begriff der Gestaltung städtischer Einschließungen, um die aktive Rolle des Staates herauszustellen, bei der vernachlässigte Viertel kein Nebenprodukt von Stadtentwicklung sind, sondern bewusst in Kauf genommen werden.

Diese These greift auch David Wilson auf. Während Wacquant eher überblicksartig bleibt, untersucht Wilson Ausgrenzung an zwei Städten des amerikanischen rust belt: Indianapolis und Cleveland. Hier zeichnet er die jüngeren Entwicklungen in benachteiligten Vierteln nach, deren Zustände er als ähnlich desaströs wie in Staaten Mittelamerikas beschreibt. Sein entscheidender Erklärungsansatz ist, dass es in Zeiten von Globalisierung und Neoliberalisierung eine besondere globale Rhetorik von Wachstum und Aufschwung die sogenannte global trope - gibt, die Städte zu einer kompetitiven Aufwertungsstrategie motiviert und sie zu Trends setzenden Laboratorien von Stadtplanern, Politikern, Architekten und Investoren macht. Es handelt sich um eine neue Form von urban governance, die die Städte regiert und die von Wilson kritisch reflektiert wird.

Nach der Lektüre der drei Beiträge im Themenheft darf somit die Frage weiter verfolgt werden, wie eine akademische und praktisch-engagierte Auseinandersetzung um sowie die Suche nach einer ,gerechten Stadt“ (Marcuse et al., 2009) gewinnbringend konzeptionalisiert werden kann.

Edited by: O. Söderström

Reviewed by: two anonymous referees

\section{Literatur}

Anderson, E.: The Code of the Streets, The Atlantic Monthly, 5, 1994.

Anderson, C. M.: Lost in Space? Ethnography and the Disparate Geographies of Social Process, The Professional Geographer 64, 176-285, 2012.

Amin, A.: The Good City, Urban Studies, 43, 1009-1023, 2006.

Bähr, J. und Mertins, G.: Die lateinamerikanische Großstadt: Verstädterungsprozess und Stadtstrukturen, Darmstadt, Wissenschaftliche Buchgesellschaft, 1995.

Berg, M.: Struktureller Rassismus oder pathologisches Sozialverhalten? Die Debatte über die Black Underclass in den USA, Herausgeber: Fluck, W. und Welf, W., in: Wie viel Ungleichheit 
verträgt die Demokratie, Armut und Reichtum in den USA, Frankfurt/New York, 47-70, 2003.

Bernt, M.: The 'Double Movements' of Neighbourhood Change: Gentrification and Public Policy in Harlem and Prenzlauer Berg, in: Urban Studies 49, 14, 3045-3062, 2012.

Bernt, M. und Holm, A.: Is it, or is not? The Conceptualisation of Gentrification and Displacement and its Political Implications in the Case of Berlin-Prenzlauer Berg, City, 13, 312-324, 2009.

Bolton, K. und Breau, S.: Growing Unequal? Changes in the Distribution of Earning across Canadian Cities, Urban Studies 49, 6, 1377-1396, 2012.

Bourdieu, P.: Die feinen Unterschiede. Kritik der gesellschaftlichen Urteilskraft, Frankfurt a. M., Suhrkamp, 1979.

Bourne, L. S.: On Schools of Thought, Comparative Research, and Inclusiveness: A Commentary, Urban Geogr., 29, 177-187, 2008.

Brailich, A., M., Germes, H., Schirmel, G., Glasze, und Pütz, R.: Die diskursive Konstitution von Großwohnsiedlungen in Frankreich, Deutschland und Polen, Europa Regional 16. H. 3, 113128, 2008.

Brenner, N., Marcuse, P., und Mayer, M.: Cities for people, not for profit, City, 13, 176-184, 2009.

Briceño-León, R. und Zubillaga, V.: Violence and Globalization in Latin America, Current Sociology, 50, 19-37, 2002.

Bromely, R. D. F. und Mackie, P. M.: Displacement and the New Spaces for Informal Trade in the Latin American City Centre, Urban Studies, 46, 1485-1506, 2009.

Bronner, K.: Grenzenlos normal? Aushandlungen von Gender aus handlungspraktischer und biografischer Perspektive (Genderstudien), Bielefeld, Transcript Verlag, 2011.

Caldeira, T. P. R.: City of Walls. Crime, Segregation, and Citizenship in São Paulo, Berkeley, University of California Press, 2000.

Castoriadis, C.: Die griechische Polis und die Schaffung der Demokratie, Herausgeber: Rödel, U., Autonome Gesellschaft und libertäre Demokratie, Frankfurt a. Main, 298-328, 1990.

Chakravorty, S.: Urban Inequality Revisited: The Determinants of Income Distribution in U.S. Metropolitan Areas, Urban Affairs Review, 31, 759-777, 1996.

Coy, M.: Gated Communities and Urban Fragmentation in Latin America: the Brazilian experience, GeoJournal, 66, 121-132, 2006.

Darden, J., Rahbar, M., Jezierski, L., Li, M., and Velie, E.: The Measurement of Neighborhood Socioeconomic Characteristics and Black and White Residential Segregation in Metropolitan Detroit: Implications for the Study of Social Disparities in Health, Ann. Assoc. Am. Geogr., 100, 137-158, 2010.

Davidson, M. und Lees, L.: New-build Gentrification: Its Histories, Trajectories, and Critical Geographies, Popul. Space Place, 16, 395-411, 2010.

Davis, D. E. und Tajbakhsh, K.: Globalization and Cities in Comparative Perspective, Int. J. Urban Regional, 29, 89-91, 2005.

Dear, M.: Comparative Urbanism, Urban Geogr., 26, 247-251, 2005.

Dear, M. und Flusty, S.: Postmodern Urbanism, Ann. Assoc. Am. Geogr., 88, 50-72, 1998.

De Certeau, M.: Kunst des Handelns, Berlin, Merve Verlag, 1988.

Deffner, V.: Habitus der Scham - Die soziale Grammatik ungleicher Raumproduktion. Eine sozialgeographische Untersuchung der Alltagswelt Favela in Salvador da Bahia (Brasilien), Passauer Schriften zur Geographie 26, Passau, 2010.

Dikeç, M.: Justice and the Spatial Imagination, Environ. Plann. A, 33, 1785-1805, 2001.

de Janvfy, A. und Sadoulet, E.: Growth, Poverty, and Inequality in Latin America: A Causal Analysis, 1970-94, Rev. Income Wealth, 46, 267-287, 2000.

de Souza, M. L.: "Phobopolis". Gewalt, Angst und soziopolitische Fragmentierung des städtischen Raumes von Rio de Janeiro, Brasilien, Geogr. Z., 100, 34-50, 2012.

Dörfler, T.: Antinomien des (neuen) Urbanismus: Henri Lefebvre, die HafenCity Hamburg und die Produktion des posturbanen Raumes- eine Forschungsskizze, Raumforschung und Raumordnung, 69, 91-104, 2011.

Dörfler, T.: Milieu und Raum - Zur relationalen Konzeptionalisierung eines sozioräumlichen Zusammenhangs, Herausgeber: Rothfuß, E. und Dörfler, T., in: Raumbezogene qualitative Sozialforschung, Wiesbaden, 33-58, 2013.

Dörfler, T. und Rothfuß, E.: Postkonstruktivismus - Jenseits von Postmoderne und cultural turn, in: Berichte, Geographie und Landeskunde, 87, 195-203, 2013.

Ehrkamp, P. und Leitner, H.: Beyond National Citizenship: Turkish Immigrants and the $(\mathrm{Re})$ Construction of Citizenship in Germany, Urban Geogr., 24, 127-146, 2003.

Fischer, K. und Parnreiter, C.: Transformation und neue Formen der Segregation in den Städten Lateinamerikas, Geogr. Helv., 57, 245-252, doi:10.5194/gh-57-245-2002, 2002.

Garbin, D. und Millington, G.: Territorial Stigma and the Politics of Resistance in a Parisian Banlieu: La Courneuve and Beyond, Urban Stud., 49, 2067-2083, 2012.

Gerhard, U.: Global City Washington, D.C. Eine politische Stadtgeographie, Bielefeld, Transcript Verlag, 2007.

Gerhard, U. und Rothfuß, E.: Armer Süden, reicher Norden? Urbane Disparitäten in einer globalisierten Welt, Praxis Geographie, 39, 40-44, 2009.

Gerhard, U.: Die Bedeutung von "Rasse" und "Klasse" im USamerikanischen Ghetto, Geographische Rundschau, 5, 18-24, 2014.

Gillette Jr., H.: Camden After the Fall: Decline and Renewal in a Post-Industrial City, Philadelphia, University of Pennsylvania Press, 2005.

Glaeser, E. L., Resseger, M., und Tobio, K.: Inequality in Cities, J. Regional Sci., 49, 617-646, 2009.

Guarnizo, L. E.: The Fluid, Multi-scalar, and Contradictory Construction of Citizenship, in: Remaking Urban Citizenship: Organizations, Institutions, and the Right to the City, Herausgeber: Smith, M. P. und McQuarrie, M., New Brunswick, NJ, Transaction Publishers, 11-35, 2012.

Hamer, J. F.: Abandoned in the Heartland: Work, Family, and Living in East St. Louis. Berkley/Los Angeles, University of California Press, 2011.

Hannerz, U.: Exploring the City: Inquiries Toward an Urban Anthropology, New York, Columbia University Press, 1980.

Harvey, D.: Social Justice and the City, Baltimore, Johns Hopkins University Press, 1973.

Harvey, D.: Justice, Nature and the Geography of Difference, Malden, MA, Blackwell, 1996.

Harvey, D.: The Right to the City, New Left Review, 53, 23-40, 2008. 
Häussermann, H., Kronauer, M., und Siebel, W.: An den Rändern der Städte: Armut und Ausgrenzung, Frankfurt a. M., Suhrkamp, 2004.

Heeg, S.: Von Stadtplanung und Immobilienwirtschaft: Die "South Boston Waterfront" als Beispiel für eine neue Strategie städtischer Baupolitik, Bielefeld, Transcript Verlag, 2008.

Holston, J.: Urban Citizenship and Globalization, Herausgeber: Scott, A. J., Global City-Regions, Trends, Theory, Policy, New York, Oxford University Press, 325-348, 2001.

Holston, J. und Appadurai, A.: Cities and Citizenship, Herausgeber: Holston, J., Cities and Citizenship, Durham, Duke University Press, 1-18, 1999.

Honneth, A.: Kampf um Anerkennung. Zur moralischen Grammatik sozialer Konflikte, Frankfurt a. M., Suhrkamp, 1992.

Isin, E. F.: Being Political: Genealogies of Citizenship, Minneapolis, University of Minnesota Press, 2002.

Jones, G. A. und Varley, A.: The Reconquest of the Historic Centre: Urban Conservation and Gentrification in Puebla, Mexico, Envoiron. Plann. A, 31, 1547-1566, 1999.

Kantor, P. und Savitch, H. V.: How to Study Comparative Urban Development Politics: A Research Note, Int. J. Urban Regional, 29, 135-151, 2005.

Kessler, S. J. und McKenna, W.: Gender. An Ethnomethodological Approach, Chicago, University Press, 2001.

Klagge, B.: "Armutsghettos" in westdeutschen Städten? Konzeptionelle Überlegungen und empirische Befunde, Die Erde, 132, 141-160, 2001.

Lapeyronnie, D.: Primitive Rebellion in the French Banlieues: On the Fall 2005 Riots, in: Frenchness and the African Diaspora: Identity and Uprising in Contemporary France, Herausgeber: Tshimanga, C., Gondola, D., und Bloom, P. J., Bloomington, Indiana, Indiana University Press, 21-46, 2009.

Lees, L.: Gentrification and Social Mixing: Towards an Inclusive Urban Renaissance, Urban Studies 45, 12, 2449-2470, 2008.

Lees, L., Slater, T., und Wyly, E. K.: Gentrification, New York, NY [u.a.], Routledge, 2008.

Lefebvre, H.: Le droit à la ville, Paris, Anthropos, 1968.

Lefebvre, H.: Kritik des Alltagslebens. Band 1 und 2. München, Hanser, 1974.

Leitner, H., Peck, J., und Sheppard, E. S. (Hrsg.): Contesting Neoliberalism: Urban Frontiers, New York/London, Guilford Press, 2007.

Lukinbeal, C., Price, P. L., und Buell, C.: Rethinking "Diversity" Through Analyzing Residential Segregation Among Hispanic in Phoenix, Miami and Chicago, The Professional Geographer, 64, 109-124, 2012.

Maffesoli, M.: Der Schatten des Dionysos, Frankfurt a. M., Syndikat, 1986.

Marcuse, H.: Vernunft und Revolution, Darmstadt/Neuwied, Luchterhand, 1972.

Marcuse, P.: Gentrification, Abandonment and Displacement: Connections, Causes and Policy Responses in New York City, Journal of Urban and Contemporary Law, 28, 195-240, 1985.

Marcuse, P., Connolly, J., Novy, J., Olivo, I., Potter, C., und Steil, J.: Searching for the Just City. Debates in Urban Theory and Practice, London/New York, Routledge, 2009.

Markus, H. R. und Moya, P. M. L. (Hrsg.): Doing Race. 21 Essays for the 21st Century, New York, W. W. Norton and Company, 2011.
Márquez, J. D.: The Black Mohicans: Representations of Everyday Violence in Postracial America, American Quaterly, 64, 625651, 2012.

Matthes, J.: The Operation called 'Vergleichen', Herausgeber: Matthes, J., in: Zwischen den Kulturen: Die Sozialwissenschaften vor dem Kulturvergleich (Soziale Welt Sonderband 8), Göttingen, 73-99, 1992.

McFarlane, C.: The Comparative City: Knowledge, Learning, Urbanism, Int. J. Urban Regional, 34, 725-742, 2010.

McFarlane, C. und Robinson, J.: Introduction - Experiments in Comparative Urbanism, Urban Geogr., 33, 765-773, 2012.

McKinnish, T., Walsh, R., und White, T. K.: Who Gentrifies Lowincome Neighboorhoods?, J. Urban Econ., 67, 180-193, 2010.

Miciukiewicz, K., Moulaert, N., Musterd, S., und Hillier, J.: Problematising Urban Social Cohesion: A Transdisciplinary Endeavour, Urban Studies, 49, 1855-1872, 2012.

Mollenkopf, J. H. und Castells, M. (Hrsg.): Dual City: Restructuring New York, New York, Russell Sage Foundation, 1991.

Murray, G.: France: The Riots and the Republic, Race Class, 47, 26-45, 2006.

Nijman, J.: Introduction - Comparative Urbanism, Urban Geogr., 28, 1-6, 2007.

Nobles, M.: "Here a Ghetto, there a Ghetto": The Value and Peril of Comparative Study, Urban Geogr., 31, 158-161, 2010.

O'Connor, A., Tilly, C., und Bobo, L. D. (Hrsg.): Urban Inequality: Evidence From Four Cities. A Volume in the Multi-city Study of Urban Inequality, New York, NY, Russell Sage Foundation, 2003.

Patillo, M.: Black Middle-Class Neighbourhoods, Annu. Rev. Sociol., 31, 305-329, 2005.

Portes, A. und Hoffmann, K.: Latin American Class Structures: Their Composition and Change during the Neoliberal Era, Lat. Am. Res. Rev., 38, 41-82, 2003.

Poster, M.: Michel de Certeau and the History of Consumerism, Diacritics, 22, 94-107, 1992.

Purcell, M.: Citizenship and the Right to the Global City: Reimagining the Capitalist World Order, Int. J. Urban Regional, 27, 564-590, 2003.

Rancière, J.: Das Unvernehmen, Frankfurt a.M., Suhrkamp, 2002.

Robinson, J.: Global and World Cities: A View from off the Map, Int. J. Urban Regional, 26, 531-554, 2002.

Robinson, J.: Ordinary Cities: Between Modernity and Development, London, Routledge, 2006.

Robinson, J.: Cities in a World of Cities: The Comparative Gesture, Int. J. Urban Regional, 35, 1-23, 2010.

Rossi, U. und A. Vanolo: Urban Political Geographies: A Global Perspective, London, Sage, 2012.

Rothfuß, E.: Exklusion im Zentrum: Die brasilianische Favela zwischen Stigmatisierung und Widerständigkeit, Bielefeld, Transcript Verlag, 2012.

Rothfuß, E: Stigmatisierung und Alltag in brasilianischen Favelas. das Beispiel Calabar in Salvador da Bahia, Geographische Rundschau, 5, 12-17, 2014.

Schmid, C.: Stadt, Raum und Gesellschaft: Henri Lefebvre und die Theorie der Produktion des Raumes, Sozialgeographische Bibliothek, Band 1. Stuttgart, Steiner, 2005.

Schmidt, R.: Soziologie der Praktiken: Konzeptionelle Studien und empirische Analysen, Frankfurt a.M., Campus, 2012. 
Schneider-Sliwa, R.: 'Hyper-Ghettos' in amerikanischen Großstädten: Lebensräume und Konstruktionsprinzip der urban underclass, Geogr. Z., 1, 27-43, 1996.

Scott, J. C.: Weapons of the Weak, New Haven/London, Yale University Press, 1985.

Slater, T.: The Eviction of Critical Perspectives from Gentrification Research, Int. J. Urban Regional, 30, 737-757, 2006.

Smith, D. M.: Geography and Social Justice, Oxford, UK, Blackwell Publishers, 1994.

Smith, M. P. und McQuarrie, M. (Hrsg.): Remaking Urban Citizenship: Organizations, Institutions, and the Right to the City. Comparative Urban and Community Research 10, New Brunswick, NJ, Transaction Publishers, 2012.

Smith, N.: The New Urban Frontier. Gentrification and the Revanchist City, London, New York, Routledge, 1996.

Soja, E.: Seeking Spatial Justice, Minneapolis/London, University of Minnesota Press, 2010.

Souza, J.: Die soziale Grammatik der peripheren Ungleichheit Für ein neues Paradigma zum Verständnis der peripheren Gesellschaften, in: Das moderne Brasilien. Gesellschaft, Politik und Kultur in der Peripherie, Herausgeber: Souza, J., Wiesbaden, 2048, 2006.

Souza, J.: Die Naturalisierung der Ungleichheit: Ein neues Paradigma zum Verständnis peripherer Gesellschaften, Wiesbaden, Springer Fachmedien, 2008.

Staeheli, L.: Cities and Citizenship, Urban Geogr., 24, 97-102, 2003.

Stren, R. E.: Urban Research in the Developing World: Latin America, Toronto, Centre for Urban und Community Studies, University of Toronto, 1994.

Varela, M. D. M. und Dhawan, N.: Mission Impossible: Postkoloniale Theorie im deutschsprachigen Raum?, Herausgeber: Reuter, J. und Villa, P.-I., in: Postkoloniale Soziologie. Empirische Befunde, theoretische Anschlüsse, politische Intervention, Bielefeld, 303-330, 2010.

Wacquant, L.: From Slavery to Mass Incarceration. Rethinking the "race question" in the US, in: New Left Review 13, January/February, 41-60, 2002.
Wacquant, L.: Roter Gürtel, Schwarzer Gürtel: Rassentrennung, Klassenungleichheit und der Staat in der französischen städtischen Peripherie und im amerikanischen Ghetto, Herausgeber: Häußermann, H., Kronauer, M., und Siebel, W., An den Rändern der Städte, Frankfurt, 184-200, 2004.

Wacquant, L.: Elias im schwarzen Ghetto, dérive, 28, 45-49, 2007.

Wacquant, L.: Urban Outcasts: A Comparative Perspective of Advanced Urban Marginality, Malden, MA, Polity, 2008a.

Wacquant, L.: Deadly Symbiosis: Race and the Rise of the Penal State, Cambridge, Polity Press, 2008b.

Wacquant, L.: The Militarization of Urban Marginality: Lessons from the Brazilian Metropolis, International Political Sociology, 2, 56-74, 2008c.

Wade, P.: Race and Ethnicity in Latin America, Chicago, Pluto Press, 1997.

Walks, A. R.: The Social Ecology of the Post-Fordist/Global City? Economic Restructuring and Socio-spatial Polarisation in the Toronto Urban Region, Urban Studies, 38, 407-447, 2001.

Ward, K.: Towards a Relationale Comparative Approach to the Study of Cities, Prog. Hum. Geogr., 34, 471-487, 2010.

Wehrhahn, R.: Urbanisierung und Stadtentwicklung in Brasilien: Aktuelle Prozesse und Probleme, Geographische Rundschau, 50, 656-663, 1998.

Wehrhahn, R.: Ghettos und Slums: begriffe, Konzepte, Diskurse, Geographische Rundschau, 5, 4-11, 2014.

Wilson, D.: Cities and Race. America's new Black Ghetto, New York, Routledge, 2007.

Wilson, D.: Racialized Poverty in the U.S. Cities: Toward a Refined Racial Economy Perspective, The Professional Geographer, 61, 139-149, 2009.

Wilson, W. J.: The Truly Disadvantaged: The Inner City, the Underclass, and Public Policy, Chicago, Univ. Pr. of Chicago, 1987.

Young, I. M.: Justice and the Politics of Difference, Princeton, Princeton University Press, 1990.

Žižek, S.: Auf verlorenem Posten, Frankfurt a.M., Suhrkamp, 2009. 